\title{
Developments of next generation refrigerants and heat transfer
}

\author{
Akio Miyara ${ }^{1,2, *}$ \\ ${ }^{1}$ Saga University, Department of Mechanical Engineering, 840-8502 Saga-shi, Japan \\ ${ }^{2}$ Kyushu University, International Institute for Carbon-Neutral Energy Research, 819-0385 Fukuoka-shi, Japan
}

\begin{abstract}
Since the effect of refrigerants which are widely used in heat pump and refrigeration systems on the global warming has become a big environmental issue, researches and developments on the next generation refrigerants are being conducted intensively. Values of global warming potential (GWP) of most of currently used refrigerants are grater than 1000. R32 which becomes popular as a refrigerant of domestics air-conditioners for years has GWP $=675$. Recently, some hydrofluoroolefins (HFOs) are getting attention and their thermophysical properties and heat transfer characteristics are being clarified experimentally because GWP of HFOs are very small in which the values of some HFOs are less than 1 and those of others are less than around 10. For example, R1234yf and R1234ze(E) are promising candidate of R134a. In this paper, recent developments of the next generation refrigerants and its heat transfer characteristics are introduced.
\end{abstract}

\section{Introduction}

In the creative period of refrigeration systems which is 1830 s, ethers, $\mathrm{CO}_{2}, \mathrm{NH}_{3}, \mathrm{SO}_{2}, \mathrm{HCs}, \mathrm{CCl}_{4}$, etc. were used as refrigerants. They are called as the first-generation refrigerants. Although ability and/or efficiency of them might be acceptable, they have defects of flammability, toxicity, very high pressure, etc. Due to the safety concerns, the use of refrigeration systems might be in limited area. For example, ice making companies and cold storage warehouse were main users.

In the 1920s, chlorofluorocarbon (CFC) and hydrochlorofluorocarbon (HCFC) which are non-flammable and nontoxic have been invented and the use of refrigeration systems was explosively expanded, such as domestic refrigerator, air-conditioner, etc. Now, our modern life is strongly supported by the refrigeration and air-conditioning technology.

However, depletion of ozone layer was found in 1980s and it was identified that the ozone layer depletion is caused by the chlorine including in the CFC and HCFC. It is well known that the usage and production of CFCs and HCFCs are regulated by the Montreal Protocol. After the Montreal Protocol, researches on alternative refrigerants were intensively conducted which are thermophysical properties, heat transfer, system performance, solubility with oil, compatibility with materials, flammability, toxicity, etc. Hydrofluorocarbons (HFCs) of which ozone depletion potential (ODP) is zero has been produced as alternative refrigerants. HFCs such as R134a, R32, R23, R410A, R404A, etc. have been successfully replaced as the refrigerants. HFCs are called as third generation refrigerants.

Another global environmental issue which is the global warming caused by increasing the concentration of carbon dioxide in atmosphere becomes a major problem and HFCs were designated as greenhouse gas by the Kyoto Protocol. The global warming potential (GWP) of most of HFCs used in refrigeration and air-conditioning fields are in the range from 1000 to 15000 . New refrigerants, hydrofluoroorefins (HFOs) which have low GWP of the order of one, were produce and measurements of thermophysical properties, heat transfer and other characteristics were started. On the other hand, use of natural refrigerants such as $\mathrm{CO}_{2}, \mathrm{NH}_{3}$, hydrocarbons, etc. is also considered as new refrigerants though they are the first generation. Some HFOs and natural refrigerants have already used in commercial machines.

In the October 2016, the HFCs were more strictly regulated by the Kigali Amendment of Montreal Protocol. For example, developed countries have to reduce $70 \%$ of the production and consumption of HFCs by 2029 . Recent abnormal weather is also said to be caused by global warming. Urgent reduction of HFCs is required. Although low GWP refrigerants have partly been used, low GWP refrigerants applicable to equipment are still limited and further developments are desired.

In this paper, the recent research on the new refrigerants are briefly overviewed and results conducted by our research group are introduced. Measurements of viscosity and thermal conductivity which are needed for heat exchanger design and simulation are reported. Some experiments on heat transfer of the next generation refrigerants are also introduced.

\footnotetext{
${ }^{*}$ Corresponding author: miyara@me.saga-u.ac.jp
} 


\section{Overview of HFOs development}

Table 1 shows the list of refrigerant candidates proposed by different companies and/or researchers for each current refrigerant. At first glance of the table, it seems to exist many candidates. However, most of the non-flammable candidates are mixture of HFC and HFO and their GWPs are not sufficiently low and there is no non-flammable candidate for R410A which is widely used in room air-conditioners, especially in Japan. $\mathrm{CO}_{2}$ is introduced as a candidate of R134a and R507 but the cycle efficiency of $\mathrm{CO}_{2}$ is low. Although R1234zd(E) and R1336mzz(Z) are nonflammable pure refrigerant of HFO, both of them are low pressure refrigerant and they cannot be candidate of high pressure refrigerants such as R134a and R410A. R290 (propane) which is a candidate of R22 used in room air conditioners and other systems has strong flammability. R1234yf and R1234ze(E) which are mildly flammability are promising candidate of R134a and they are already used in actual machines. Mildly flammable refrigerants have been accepted in some country such as Japan. The GWP of mildly flammable candidate is lower than non-flammable candidates but they cannot overcome the regulation by the Kigali Amendment. We have to find new candidates of refrigerants especially for R404A, R507, R410A, and R22.

Table 1. Proposed refrigerant candidates of each current refrigerants.

\begin{tabular}{|c|c|c|c|}
\hline $\begin{array}{c}\text { Current } \\
\text { Refrigerants }\end{array}$ & Non-Flammable candidates & Mildly Flammable candidates & Flammable candidates \\
\hline $\mathrm{R} 134 \mathrm{a}$ & $\begin{array}{l}\text { R450A, R513A (HFC/HFO) } \\
\text { R744 (CO2) }\end{array}$ & $\begin{array}{l}\text { R516 (HC/HFO) } \\
\text { R1234yf, R1234ze(E) }\end{array}$ & $\begin{array}{l}\text { R290 (propane) } \\
\text { R600a (iso-butane) }\end{array}$ \\
\hline $\begin{array}{l}\text { R404A } \\
\text { R507 }\end{array}$ & $\begin{array}{l}\text { R448A, R449A/B (HFC/HFO) } \\
\text { R452A, R452C (HFC/HFO) } \\
\text { R744 (CO2) }\end{array}$ & $\begin{array}{l}\text { R465A (HFC/HFO) } \\
\text { R457A (HFC/HFO) } \\
\text { R455A, R454C (HFC/HFO) } \\
\text { R717 (ammonia) }\end{array}$ & N/A \\
\hline R410A & N/A & $\begin{array}{l}\text { R32 (HFC) } \\
\text { R459A, R447A (HFC/HFO) } \\
\text { R452B, R454B (HFC/HFO) }\end{array}$ & N/A \\
\hline R123 & $\begin{array}{l}\text { R1234zd(E) (HFO) } \\
\text { R514A } \\
\text { (R1336mzz/dichloroethane) } \\
\text { R1336mzz(Z) (HFO) }\end{array}$ & (5) & N/A \\
\hline $\mathrm{R} 22$ & R448A R449A/B (HFC/HFO) & R457A, R454C (HFC/HFO) & R290 (propane) \\
\hline
\end{tabular}

\section{Thermophysical properties of HFOs}

Thermophysical properties are divided two categories, thermodynamic properties and transport properties. The thermodynamic properties which are critical point, saturation pressure, pressure-temperature-density relation, and sound speed of new refrigerants are needed to obtain the equation of state by which enthalpy, entropy, and other thermodynamic properties can be calculated. And, cycle performance of refrigerants can be estimated by the obtained values. Appropriate measurements of R1234yf, R1234ze(E), R1233zd(E), and R1336mzz(E) have been done by other researchers [e.g. 1-4]. The transport properties which are viscosity, thermal conductivity, and diffusivity were measured by our laboratory and some other researchers [e.g. 5] though the number of open reports is very limited. The transport properties are needed for design of heat exchanger, cycle simulation, etc.

In the next section, measurements of viscosity and thermal conductivity are introduced, and importance of accurate value is explained.

\subsection{Viscosity}

Viscosity of refrigerants was measured by tandem capillary tube method which is originally proposed by our laboratory [6].

The tandem capillary tube method is based on Hagen-Poiseuille equation and is a system to determine the viscosity of test fluids from a measured value of the pressure drop of long and short capillary tubes in a laminar flow. The relationship between viscosity $\eta$ and the pressure drop $\Delta P$ of a fluid that flows with flow rate of $q$ through a horizontal tube with radius $a$ and length $L$ were expressed by the equation of Hagen-Poiseuille as,

$$
\eta=\frac{\pi a^{4} \Delta P}{8 L q}
$$


The pressure drop is usually measured at chambers installed in inlet and outlet of the capillary tube where the inlet/outlet losses should be considered and the relation between viscosity and pressure drop can be correlated with the following equation with equipment parameters $m$ and $n$.

$$
\eta=\frac{\pi a^{4} \Delta P}{8 q(L+n a)}-\frac{m \rho q}{8 \pi(L+n a)}
$$

In the proposed tandem capillary tube method shown in Fig.1, the end effects are cancelled and the viscosity can be calculated by using the following equation which has no equipment parameter.

$$
\eta=\frac{\pi\left(a_{l}^{4} \Delta P_{l}-a_{s}{ }^{4} \Delta P_{s}\right)}{8 q\left(L_{l}-L_{s}\right)}
$$

Where, subscripts $l$ and $s$ indicate long and short tube, respectively.

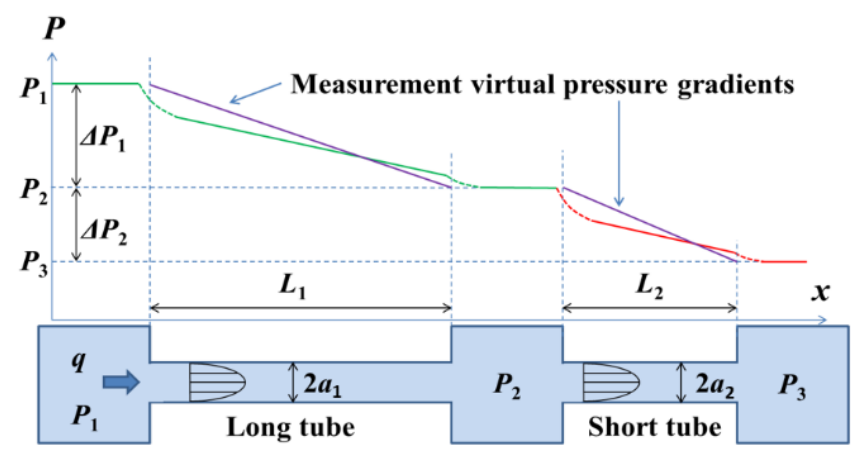

Fig. 1. Concept of tandem capillary tube method.

As an example, measured viscosities of R1233zd(E) in liquid phase are shown in Fig.1. The measurement was carried out in the temperature of 314 to $434 \mathrm{~K}$ and pressure of 1.0 to $4.07 \mathrm{MPa}$. Similarly with typical trend, viscosity is a strong function of density. Increasing the temperature causes decreasing density and then viscosity decreases. The effect of pressure is not so strong. In the figure, calculated results by REFPROP ver.9.1 [7] in which the estimation was adjusted to fit with other experimental data provided by Hulaw et al. [8]. There are large discrepancies between our data and value calculated by REFPROP ver.9.1. This discrepancy is not acceptable for design of heat exchanger and etc.

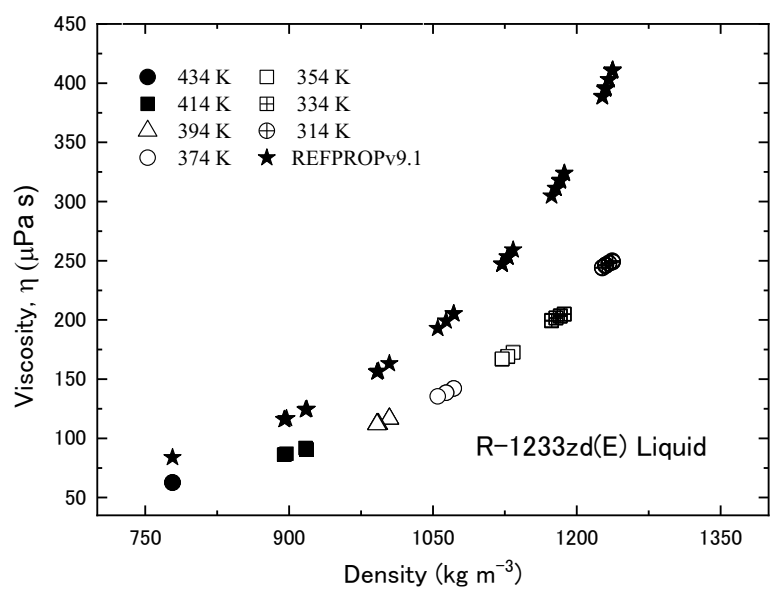

Fig. 2. Comparison of measured viscosity of R1233zd(E) in liquid phase and calculated values of REFPROP ver.9.1. 
In generally, it is not easy to judge which measurement value is correct in thermophysical property measurements. However, reliability of our data was able to be proved by measured condensation heat transfer coefficient by Nagata et al. [9] where the experiments were carried out in the range of saturation temperature of $293.15 \mathrm{~K}$ and $313.15 \mathrm{~K}$. The dotted and solid lines present the theoretically calculated heat transfer coefficient by Nusselt theory [10] with the viscosity values of REFPROP ver.9.1 and our data. As shown in figure, the experimental heat transfer coefficients agree well with Nusselt theory used our measured viscosity. On the other hand, calculated values used REFPROP ver.9.1 are much lower than the experimental data. Experimental condensation heat transfer coefficients of other refrigerants agreed with Nusselt theory. This results imply the confirmation of the reliability of present experimental viscosity data of R-1233zd(E) [11]. In other words, correct values of thermophysical properties are necessary for estimation of heat transfer coefficients and for design of heat exchanger.

In the REFPROP ver.10.0 which is an updated new version, our data were used to adjust calculated values.

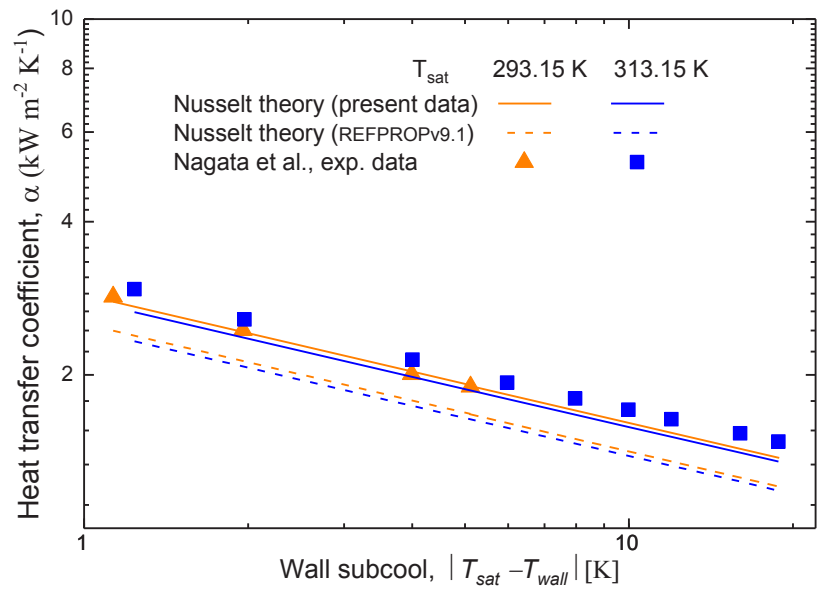

Fig. 3. Viscosity of liquid R-1233zd(E) as a function of density.

\subsection{Thermal conductivity}

The thermal conductivity of new refrigerants were measured by transient hot wire method. In the case of ideal model where an infinity-long line heat source with sufficiently small diameter, after step-wise heating $Q$ the transient temperature variation $\Delta T$ of the line heat source immersed in a fluid is expressed by the following equation.

$$
\Delta T(t, r)=\frac{Q}{4 \pi \lambda} \ln \frac{4 \alpha t}{r^{2} C}
$$

Where, $\lambda$ is thermal conductivity, $t$ is time, $\alpha$ is thermal diffusivity, $r$ is radius, and $C$ is a constant. Differentiation of this equation with $\ln t$ gives

$$
\lambda=\frac{Q}{4 \pi(d \Delta T / d \ln t)}
$$

Eq.(5) is a basic equation to calculate the thermal conductivity from experimental data which are heating rate and temperature change in time.

Fig. 4 shows a schematic view of thermal conductivity measurement equipment $[12,13]$. In other to realize the ideal condition, long and short platinum wires with diameter $15 \mu \mathrm{m}$ were installed in pressure vessel. By using the long and short wires, end effects at the connection point of the platinum wire can be eliminated. The pressure vessel is set in the room covered by electric heater to keep a uniform temperature condition. The test fluids is pressurized by Helium gas and the pressure in the test vessel is controlled to a desired value. In order to measure the temperature variation of the platinum wires, the long and short wires were installed in a Wheatstone bridge circuit consist of standard resistances.

Measured thermal conductivities of R1233ze(E) in liquid phase are shown in Fig.5. The liquid thermal conductivity was measured in the range of temperature of 313 to $433 \mathrm{~K}$ and pressure of 1.0 to $4.0 \mathrm{MPa}$. The values of measured thermal conductivity agree well with calculated value of REFPROP ver.9.1 in which data obtained by Perkins et al. [5] were used to adjust the estimation values. This result is largely different from the result of viscosity. 


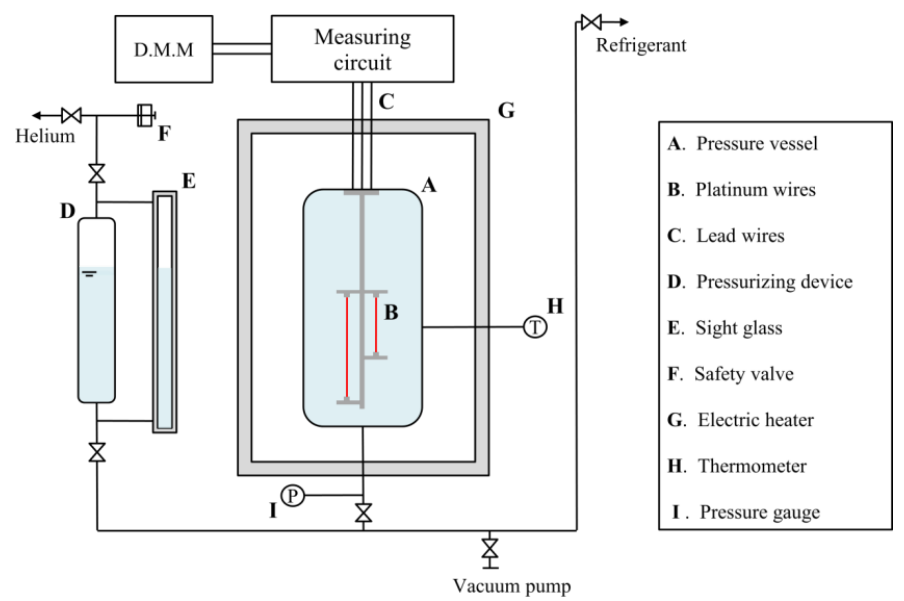

Fig. 4. Schematic view of thermal conductivity measurement equipment.

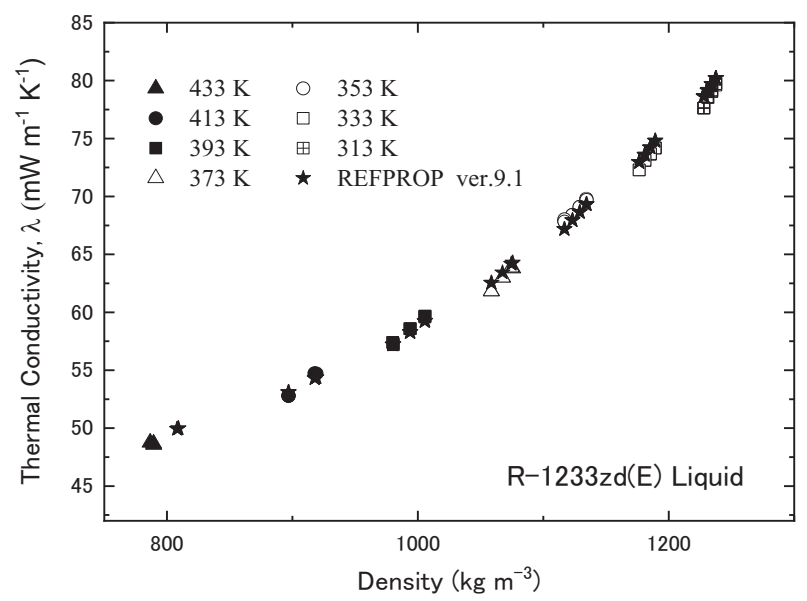

Fig. 5. Comparison of measured viscosity of R1233zd(E) in liquid phase and calculated values of REFPROP ver.9.1.

\section{Heat transfer characteristics of $\mathrm{HFO}$ and $\mathrm{HFC} / \mathrm{HFO}$ mixture}

As a typical result, in-tube condensation heat transfer characteristics [14] are explained.

Fig. 6 shows schematic of test section used to measure the in tube heat transfer coefficient. The test section is a horizontally installed double tube heat exchanger with a length of $3.6 \mathrm{~m}$. Refrigerant flows inside an inner tube and cooling water flows through the annular space in counter current. The annular channel is divided into 12 subsections where effective heat transfer length of the subsection is $300 \mathrm{~mm}$. The inner test tube is made of smooth copper tube of $4.35 \mathrm{~mm}$ inner diameter and $6.35 \mathrm{~mm}$ outer diameter and the outer tube is made of polycarbonate resin pipe of $9 \mathrm{~mm}$ inner diameter and $13 \mathrm{~mm}$ outer diameter. Mixing cup temperature of cooling water is measured at inlet/outlet of each subsection. At the central position of each subsection the outside wall temperature of the inner tube is measured with four copper constantan thermocouples which are distributed circumferentially at the top, left, bottom and right sides of the tube.

Heat transfer coefficients of R1234ze(E), R32, R410A, and mixture of R1234ze(E) and R32 are shown in Fig.7. Condensation temperature is $40{ }^{\circ} \mathrm{C}$ and two different mass flux conditions, 300 and $400 \mathrm{~kg} \mathrm{~m}^{-2} \mathrm{~s}^{-1}$, are indicated. (1-x) is wetness which is a ratio of liquid mass flow rate and total mass flow rate. The heat transfer coefficient decreases as the condensation progresses for both pure and mixture refrigerants. Both the mixtures show lower heat transfer coefficient than the pure R1234ze(E) and R32. When condensation occurs at the papor-liquid interface of the mixture the temperature at the interface is always less than the temperature of the axial papor flow, so that the mass fraction of more volatile component (R32) at the interfacial papor is always greater than the mass fraction of the bulk axial papor flow. A 
counter diffusion of more volatile component (R32) from the interface to the bulk papor flows occurs, which retards the mass diffusion from the papor core to the interface and results in a reduction of the condensation and thus the heat transfer rate. This mass transfer resistance also causes decrease of papor-liquid interface temperature and heat transfer is degraded. Moreover, the higher liquid thermal conductivity of pure R32 is responsible for its higher heat transfer coefficient than the mixture.

The heat transfer coefficient of 70\% R1234ze(E) shows lower heat transfer coefficient than that of 55\% R1234ze(E) mixture because the $70 \% \mathrm{R} 1234 \mathrm{ze}(\mathrm{E})$ mixture has lower liquid thermal conductivity than the 55\% R1234ze (E) mixture at same saturation temperature[15]. By comparing R410A and both the mixture, it is known that the condensation heat transfer coefficients of the mixture with $55 \% \mathrm{R} 1234 \mathrm{ze}(\mathrm{E})$ are comparable with R410A though that of $70 \% \mathrm{R} 1234 \mathrm{ze}(\mathrm{E})$ is somewhat lower. This fact implies that a mixture with suitable concentration can be a promising candidate of R410A although it is only a view from heat transfer performance.

The results shown in Fig. 7 also shows that the pure refrigerants R1234ze(E), R32, R410A and the mixtures of $\mathrm{R} 1234 \mathrm{ze}(\mathrm{E})$ and R32 exhibit high heat transfer coefficient at the high mass flux condition. This is due to the thinning of liquid film on the tube surface at the high mass flux condition. For the mixture, the effect of the mass transfer resistance due to the composition and temperature change in the vapor phase decreases as the vapor velocity increases.
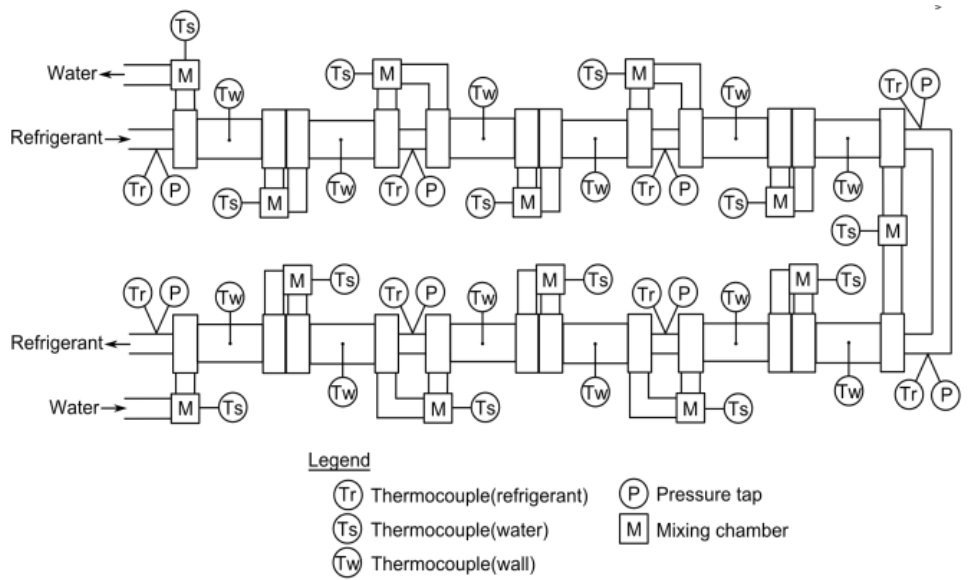

Fig. 6. Test section to measure in-tube heat transfer coefficient.

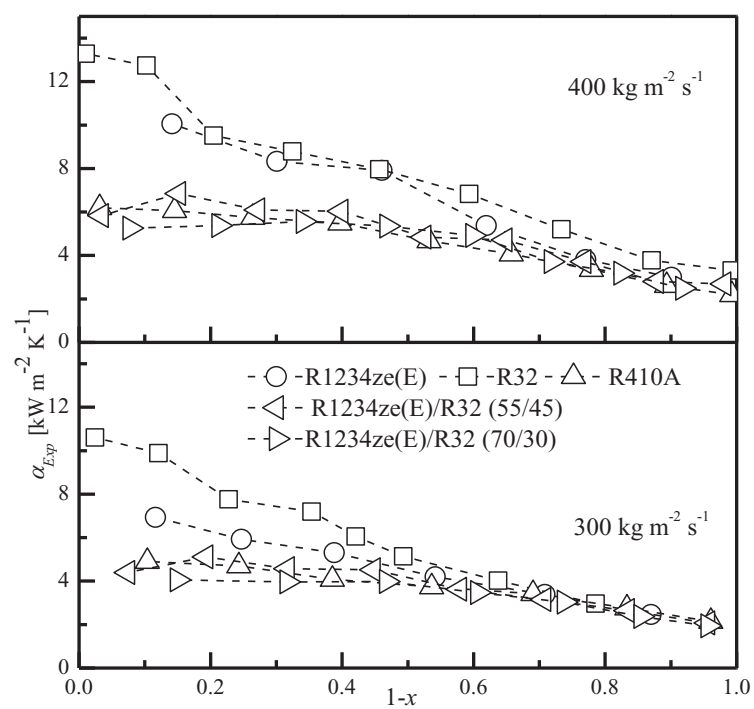

Fig. 7. Experimental condensation heat transfer coefficient distribution with wetness. 


\section{Conclusions}

Recent developments of next generation refrigerants were briefly introduced and some measurement results on thermophysical properties and heat transfer of the new refrigerants were shown. Although there are some promising candidates in the application field of low pressure refrigerant used in turbo chiller and high temperature heat pump, promising candidates of high pressure refrigerants have not found yet. Because pure refrigerants of next generation refrigerant candidate are very limited, to find suitable combinations and to determine its optimum concentrations is also needed.

Prior to other studies, thermophysical properties have to be quickly measured and equation of state, correlations of transport properties should be derived for the next step of developments such as heat transfer experiment, system performance test, system simulation, etc. To equip the equation of state and correlations in to a computer software like REPROP is desired. And, heat transfer characteristics and capacity of refrigerant candidates should be clarified experimentally. Development of heat transfer enhancement technique especially for mixtures are required.

Although it was not mentioned in the previous chapters, system performance tests by experimental and numerical methods are needed for the selection of refrigerant combination and concentration by using experimental method and numerical simulation.

Prompt and further researches are needed for overcome the world regulation and preserve the earth.

\section{References}

1. M. Richter, M.O. McLinden, E.W. Lemmon, J. Chem. Eng. Data, 56(7), 3254-3264 (2011).

2. M. Thol, E.W. Lemmon, Int. J. Thermophys., 37, 28 (2016).

3. R. Akasaka, E.W. Lemmon, Proc. 8th Asian Conference on Refrigeration and Air Conditioning, Taipei (2016).

4. G. Di Nicola, L. Fedele, J.S, Brown, S. Bobbo, G. Coccia, J. Chem. Eng. Data, 62(9), 2496-2500 (2017).

5. R.A. Perkins, M.L. Huber, M.J. Assael, J. Chem. Eng. Data. 62 (9), 2659-2665 (2017).

6. Md J. Alam, A. Miyara, K. Kariya, K.K. Kontomaris, J. Chem. Eng. Data 63(5), 1706-1712 (2018).

7. E.W. Lemmon, M.L. Huber, M.O. McLinden, NIST REFPROP ver. 9.1; (2015).

8. R.J. Hulse, R.S. Basu, R.R. Singh, R.H.P Thomas, J. Chem. Eng. Data. 57 (12), 3581- 3586 (2012).

9. R. Nagata, C. Kondou, S. Koyama, Int. J. Refrig. 63, 157-170 (2016).

10. W. Nusselt, Zeitschrift des Verein Deutscher Ingenieure 60 (27), 541-546 (1916).

11. A. Miyara, Md. J. Alam, K. Kariya, Int. J. Refrig. 92, 86-93 (2018).

12. Md.J. Alam, M.A. Islam, K. Kariya, A. Miyara, Int. J. Refrig. 84, 220-227 (2017).

13. Md.J. Alam, M.A. Islam, K. Kariya, A. Miyara, Int. J. Refrig. 90, 74-180 (2018).

14. A. Miyara, H.M.M. Afroz, Md. A. Hossain, Proc.15th International Heat Transfer Conference, Kyoto, (2014).

15. A. Miyara, K. Tsubaki, N. Sato, R. Fukuda, Proc. 23rd IIR Int. Con. Refrig., Prague, (2011). 